\title{
Cutaneous Manifestation of COVID-19: A Short Clinical Case
}

\section{RATESH BASSI ${ }^{* 1}$, GEETIKA BASSI ${ }^{2}$}

Much is now known about the respiratory presentation of coronavirus SARS-CoV-2 (COVID-19) but it can also show up with clinical manifestations in other locations, such as on the skin. Herein, we describe a case with cutaneous symptoms that emerged during the recovery phase in a SARS-CoV-2 coronavirus infected patient. It is important for the healthcare professionals as well as the patients to know about such scenarios, so that appropriate action can be readily taken.

KEYWORDS: Coronavirus, SARS-CoV-2, COVID-19

\section{INTRODUCTION}

The disease caused by the novel coronavirus SARS-CoV-2 (COVID- 19) was first described in China in December 2019 and is characterized by the appearance of symptoms such as fever, dry cough, dyspnoea, rhinorrhoea, anosmia, and ageusia. The horizon of respiratory involvement ranges from an upper airway catarrh, that can go unnoticed, even severe pneumonia or syndrome acute respiratory illness due to coronavirus. ${ }^{1}$ Although less frequently, dermatological manifestations have been described in association with COVID-19. In a study conducted by Recalcati in a sample of 88 cases, it was found that 18 patients had cutaneous manifestations, including 3 with generalized urticaria, 14 cases of erythematous rash, and one with varicelliform rash. $^{2}$ These skin symptoms are not specific to COVID-19 and have characteristics similar to those that occur in other respiratory infections with common viral causes.

\section{CASE REPORT}

We present the case of a 49-year-old man with no relevant medical history who presented to the emergency room with dry cough and dyspnea of moderate efforts for 7 days. By profession, the subject was a taxi driver but denied any recent contact with tourists or passengers due to lockdown. The subject also denied use of alcohol and tobacco and was not on any long-term medication. No chronic comorbidities were evident. On physical examination, the patient was afebrile, and pulmonary auscultation was normal.
He was able to pass air in and out of the lungs normally. Chest $\mathrm{x}$-ray revealed peripheral and bilateral pulmonary opacities, predominantly in the lower lobes. The observed signs and symptoms were compatible with COVID-19. The polymerase chain reaction test for SARS-CoV-2 was done which came out to be positive. He was admitted and treatment with hydroxychloroquine and lopinavir/ritonavir was initiated. The subject showed clinical improvement and polymerase chain reaction for coronavirus SARS-CoV-2 gave negative result after two weeks. The subject was discharged but second day after discharge, he presented again with slightly itchy erythematous maculopapular rash with islets of healthy skin on the trunk, which appeared suddenly. Topical betamethasone ointment was prescribed along with oral levocetirizine, but after few hours, the lesions extended to cervical region, face and proximal region of upper limbs, oral prednisone was added to his treatment regimen. The clinical response was favorable, with disappearance of lesions in a few days.

\section{DISCUSSION}

Cutaneous symptoms are not too common in COVID-19 cases and in most patients appear after hospital discharge, as in our case, and are not associated with increased severity of COVID-19. In a previously conducted study, the skin lesions in COVID-19 patients mainly affected the trunk, were asymptomatic or slightly pruritic and then disappeared in a few days. ${ }^{2}$ Another previously 
published case of COVID-19 was initially mistaken for dengue due to the similarity in cutaneous manifestations. ${ }^{3} \quad$ Other dermatological manifestations to consider are those due to side effects of certain drugs used in the treatment of COVID-19. In particular, one must be aware of the possible adverse effects of hydroxychloroquine on the skin such as skin hyperpigmentation, pruritus, xerosis, alopecia, urticaria, morbilliform rashes or maculopapular and exfoliative dermatitis. ${ }^{4}$ Azithromycin can also cause skin rashes, itching or Stevens-Johnson syndrome. The most common adverse effects of the drug lopinavir/ritonavir are maculopapular rashes, pruritus, eczema and seborrheic dermatitis. Micro-thrombosis related to endothelial damage and vascular disorders in COVID-19 may also lead to other more serious skin lesions. They are ischemic lesions of sudden onset, characterized by cyanosis, blistering and dry gangrene of the fingers and toes. ${ }^{5}$

\section{CONCLUSION}

It is evident from the literature that COVID-19 can lead to skin manifestations, more studies are needed to know all the forms of presentation and confirm the causal relationship with coronavirus infection. It is important to rule out all other causes of skin lesions in COVID-19 affected subjects. For timely appropriate action, regular follow up of such patients should be scheduled for sure.

\section{REFERENCES}

1. Wang D, Hu B, Hu C, Zhu F, Liu X, Zhang J, et al. Clinical characteristics of 138 hospitalized patients with 2019 novel coronavirus-infected pneumonia in Wuhan, China. JAMA. 2020;323:1061-9.

2. Recalcati S. Cutaneous manifestations in COVID-19: A first perspective. J Eur Acad Dermatol Venereol. 2020;34(5):e212-e213.

3. Joob B, Wiwanitkit V. COVID-19 can present with a rash and be mistaken for dengue. J Am Acad Dermatol. 2020 May;82(5):e177.

4. Fernandez AP. Updated recommendations on the use of hydroxychloroquine in dermatologic practice. J Am Acad Dermatol. 2017;76:1176-82.

5. Zhang Y, Cao W, Xiao M, Li YJ, Yang Y, Zhao J, et al. Clinical and coagulation characteristics of 7 patients with critical COVID-2019 pneumonia and acro-ischemia. Zhonghua Xue Ye Xue Za Zhi. 2020;41(o):Eoo6.

Source of support: Nil, Conflict of interest: None declared 